\title{
Post-amputation pain among lower limb amputees in a tertiary care hospital in Jeddah, Saudi Arabia
}

\author{
A retrospective study
}

Dareen A. AlMehman, MBBS, SBA, Abrar S. Faden, MBBS, Badr M. Aldahlawi, MBBS, Mohammed S. Bafail, MBBS, Maram T. Alkhatieb, MBBS, SBA, Abdullah M. Kaki, MBCbB, FRCPC.

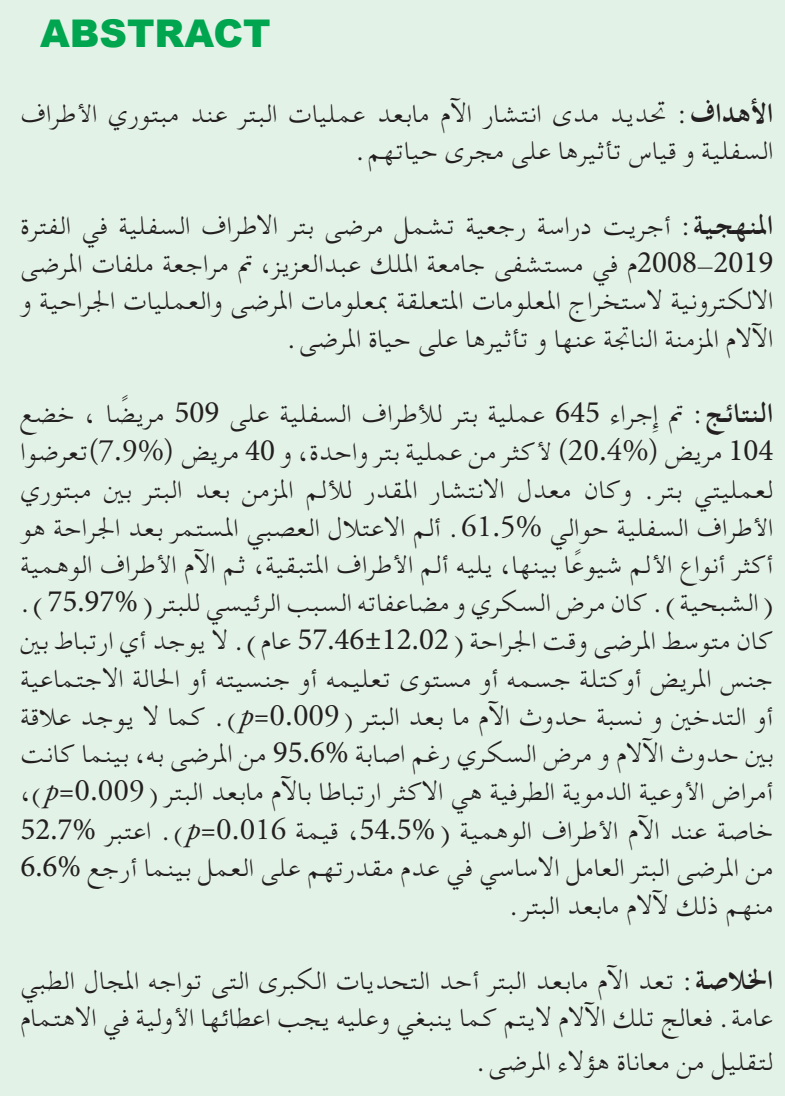

Objectives: To estimate the prevalence of postamputation pain among lower-limb amputees and its burden on their lives.

Methods: A retrospective patients record review of lower limb amputation surgeries was carried out at King Abdulaziz University Hospital, Jeddah, Saudi Arabia, between 2008-2019. e collected data included: patients demographics, surgical operations, chronic pain characteristics, functional impairment, and treatment.

Results: A total of 645 lower-limb amputations carried out on 509 patients, 104 (20.4\%) patients had more than one amputation surgery carried out, and 40 (7.9\%) patients having bilateral amputations. The estimated prevalence of chronic post-amputation pain among the lower-limb amputees was approximately $61.5 \%$. Persistent postoperative neuropathic pain was the most common type of pain following amputation, followed by residual limb pain, then phantom limb pain. The most common cause of amputation was diabetes and its related complications (76\%). The mean age at surgery was $57.46 \pm 12.02$ years. Although diabetes was a common comorbid illness (95.6\%), it had no correlation with the development of chronic post-amputation pain. However, peripheral vascular disease had a strong association $(p=0.009)$, especially with the development of phantom limb pain $(54.5 \%$; $p=0.016$ ). The most common reason for functional impairment among the amputees was disability $(52.7 \%)$, while only $6.6 \%$ said it was secondary to pain.

Conclusion: Post-amputation pain is one of the major consequences of amputation. Pain was poorly managed among these patients. Special considerations should be obtained to manage these patients' pain and reduce their suffering.

Keywords: lower-limb amputation, post-amputation pain, phantom pain, stump pain, residual limb pain, persistent postoperative neuropathic pain

Saudi Med J 2022; Vol. 43 (2): 187-196 doi: 10.15537/smj.2022.43.2.20210609

From the Department of Anesthesia (AlMehman, Aldablawi), King Fahd Armed Forces Hospital, from the Department of Anesthesia (Faden), from the Department of Surgery (Alkhatieb), King Abdulaziz University Hospital, from the Department of Anesthesia (Bafail), King Abdulaziz Medical City; and from the Department of Anesthesia and Critical Care (Kaki), Faculty of Medicine, King Abdulaziz University, Jeddah, Kingdom of Saudi Arabia.

Received 4th August 2021. Accepted 7th December 2021

Address correspondence and reprint request to: Dr. Abdullah M. Kaki, Professor and Consultant of Anesthesiology and Pain Management, Department of Anaesthesia and Critical Care, Faculty of Medicine, King Abdulaziz University, Jeddah, Kingdom of Saudi Arabia.E-mail:akaki@kau.edu.sa ORCID: https://orcid.org/0000-0002-0584-0987 
$A^{n}$ mputation has been known since the dawn of mankind. Post-amputation pain (PAP) is one of the major consequences of amputation that challenge pain physicians. ${ }^{1}$ Limb amputation is only carried out when no other possible treatment option will save a patient's life. Limb amputations are carried out for various reasons, ranging from vascular diseases, serious infections, tumors, and traumas to congenital malformations. A meta-analysis showed that more than $50 \%$ of amputations were due to diabetes or vascular etiologies. Vascular insufficiency amputees have a $27-44 \%$ risk of contralateral limb amputation after 4 years. $^{2-5}$

Diabetic patients are more exposed to complications that might lead to lower extremity amputation than are nondiabetic individuals. With the prevalence of type 2 diabetes in Saudi Arabia ranging from 18.2\% in the Eastern province to $31.6 \%$ in the Central region, the rate of amputation increases significantly. ${ }^{6}$

Limb amputation can lead to painful and non-painful conditions. The painful consequences can be classified as phantom sensation, phantom limb pain (PLP), and residual limb pain (RLP; previously known as stump pain). ${ }^{5,7}$ Phantom pain is the perception of unpleasant sensations in the nerve distribution of the missing body part. It has been reported to occur in $47-79 \%$ of post-amputation patients. ${ }^{7-14}$ Residual limb (stump) pain is pain localized in the residual body part following amputation. Recent studies have reported an incidence of RLP of 32-93\% with long-duration follow-up. ${ }^{7-11,14-16}$

There are many hypotheses on the development of PAP among amputees, but most of them fail to refer a certain mechanism or factor. However, many experts believed that there are 3 major mechanisms that might cause these phenomena: the peripheral mechanism, the central neural mechanism, and the psychogenic mechanism. ${ }^{17}$

The aims of this study were to estimate the incidence of post-amputation pain among individuals who have had lower-limb amputation surgeries in a sample of local residents; determine the effect of sociodemographic factors, habits, and illnesses on the development and the severity of PAP; find any correlation between preoperative pain and the development of PAP; and identify the characteristics of such pain.

Disclosure. Authors have no conflict of interests, and the work was not supported or funded by any drug company.
Methods. The medical records of 509 patients who underwent lower-limb amputation in the period between January 2008 and December 2019 were identified from the hospital information system after the approval of the Ethics and Research Committee at King Abdulaziz University Hospital, Jeddah, Saudi Arabia (Reference no 85-19) on February, 2019. Data related to amputation, phantom limb sensation, and post-amputation pain were recorded. The study was carried out in accordance with the principles stated in the Declaration of Helsinki.

All adult patients who underwent lower-limb amputations and were aged 18 years or older at the time of surgery were included in the study. Perioperative variables were recorded, including demographic data, associated medical diseases (comorbidities), body mass index (BMI), surgical data, reason for amputation, level and side of amputation, intraoperative nerve ligation, type of anesthesia used, pre- and post-amputation pain, used medications, physical therapy consultation, and referral to other specialties.

We carried out a phone call interview with each subject to assess the occurrence of phantom limb or pain, persistent postoperative pain, residual limb pain, and any neuropathic pain. One of the research team completed a 6-page questionnaire adapted and modi ed from Kooijman et $\mathrm{al}^{18}$ phantom pain questionnaire, Wartan et $\mathrm{al}^{8}$ phantom pain sensation 4-page amputee questionnaire, and a modified DN4 questionnaire. ${ }^{19}$ The physical examination part of each questionnaire was not included in the interviews. Each patient's agreement to complete the questionnaire was considered a consent to participate in the study. Patients were excluded from the study if they refused to participate in the study.

The study questionnaire covered various aspect of the patient's life: sociodemographic data, functional assessment, smoking habits, the presence of pain and its duration before the amputation, the complaint of residual limb pain, phantom limb pain, or persistent postoperative pain, the characteristics and the severity of pain, and its effect on daily activities, treatment options, and surgical data.

On an 11-point numerical scale, pain was assessed by asking the patient to rate the severity of their pain, where 0 was no pain and 10 was the worst imaginable pain. Participants were also asked if they had ever consulted a physician regarding their pain, treatment modalities they had received, their satisfaction with the treatments provided, and how they cope with their pain.

Statistical analysis. Data was entered into a Microsoft Office Excel spreadsheet. Statistical analysis was carried out using Statistical Package for Social Sciences for 
Windows, version 22 (IBM Corp., Armonk, NY, USA). Numerical variables were presented as mean and standard deviation, or frequency and percentage. The Chi-square test was used to test the independence of row and column variables, without indicating the strength or direction of the relationship. Pearson Chi-square and likelihood-ratio Chi-square, Fisher's exact test, and Yates' corrected Chi-square were computed for $2 \times 2$ tables. The prevalences of post-amputation pain syndromes among all lower-limb amputees were calculated. The numbers of specific pain-syndrome patients were divided by the total number of amputees and then multiplied by 100 to find the percentage. $P$-value of $<0.05$ were considered significant.

Results. A total of 645 lower-limb amputation surgeries were carried out at King Abdulaziz University Hospital, Jeddah, Saudi Arabia, including: 2 (0.3\%) hip disarticulations, 271 (42\%) above-knee amputations (AKA), 230 (35.7\%) below-knee amputations (BKA), 21 (3.3\%) foot amputations, 33 (5.1\%) forefoot amputations, and $88(13.6 \%)$ toe amputations from January 2008 until December 2019. Of the 509 patients who underwent the 645 amputation surgeries, $104(20.4 \%)$ patients had more than one amputation surgery, and $40(7.9 \%)$ had bilateral amputations. e most likely reason for amputation was related to diabetes complications and diabetic foot (76\%).

Each one of the 645 amputation surgeries was considered as an individual patient. The mean age at surgery was $57.46 \pm 12.02$ years, and 458 (71\%) patients were male. There were 198 surgeries carried out on Saudis (30.7\%) and 447 on non-Saudis $(69.3 \%$; Table 1).

At the time of the interview, 276 patients had died, 258 patients were uncontactable, and 111 patients were alive and agreed to participate in the study. There were 20 patients subsequently excluded from the final analysis because they had multiple amputation surgeries carried out on the same limb. The final level of amputation was included in the research. A total of 91 cases were finally included in the study (Figure 1).

The patients' age range was 34-85 years, with a mean age of $57.46 \pm 12.022$ year. Of those, $56(61.5 \%)$ patients were suffering from post-amputation pain, 22 (24.2\%) experienced PLP, 28 (30.8\%) experienced RLP, and $33(36.3 \%)$ had symptoms suggestive of persistent postoperative neuropathic pain. A total of 35 (38.5\%) were pain free, $14(15.4 \%)$ had phantom pain without RLP, and 8 (8.8\%) had both PLP and RLP concomitantly. There was no significant association found between PLP and RLP $\left(\chi^{2}=0.418 ; p=0.518\right)$.
Table 1 - Patients demographic data (N=509).

\begin{tabular}{|c|c|c|}
\hline Variables & n & $\%$ \\
\hline \multicolumn{3}{|l|}{ Age group (years) } \\
\hline$<30$ & 5 & 0.78 \\
\hline $31-40$ & 28 & 4.34 \\
\hline $41-50$ & 72 & 11.16 \\
\hline $51-60$ & 179 & 27.75 \\
\hline $61-70$ & 216 & 33.49 \\
\hline$>70$ & 41 & 6.36 \\
\hline Mean \pm SD & \multicolumn{2}{|c|}{$57.46 \pm 12.02$} \\
\hline \multicolumn{3}{|l|}{ Gender } \\
\hline Female & 187 & 28.99 \\
\hline Male & 458 & 71.01 \\
\hline \multicolumn{3}{|l|}{ Nationality } \\
\hline Saudi & 198 & 30.70 \\
\hline Non-Saudi & 447 & 69.30 \\
\hline \multicolumn{3}{|l|}{ Post-amputation pain } \\
\hline No Pain & 35 & 38.5 \\
\hline Complain of pain & 56 & 61.54 \\
\hline Persistent neuropathic pain & 14 & 15.4 \\
\hline Residual limb pain & 9 & 9.9 \\
\hline Phantom pain & 10 & 11.0 \\
\hline $\begin{array}{l}\text { Persistent neuropathic pain+ residual limb } \\
\text { pain }\end{array}$ & 11 & 12.1 \\
\hline $\begin{array}{l}\text { Persistent neuropathic pain + phantom } \\
\text { Pain }\end{array}$ & 4 & 4.4 \\
\hline Residual limb pain + phantom pain & 4 & 4.4 \\
\hline All types & 4 & 4.4 \\
\hline \multicolumn{3}{|l|}{ Number of amputation surgeries per patient } \\
\hline Once & 405 & 79.57 \\
\hline More than 1 & 104 & 20.43 \\
\hline Bilateral & 40 & 7.86 \\
\hline \multicolumn{3}{|l|}{ Reasons for amputation } \\
\hline Peripheral vascular disease (PVD) & 47 & 7.29 \\
\hline Thromboembolism / acute limb ischemia & 19 & 2.95 \\
\hline Diabetes complications / diabetic foot & 490 & 75.97 \\
\hline Infection & 73 & 11.32 \\
\hline Trauma & 8 & 1.24 \\
\hline Cancer & 5 & 0.78 \\
\hline Other & 3 & 0.47 \\
\hline
\end{tabular}

Patient's age was significantly associated with the development of chronic post-amputation pain $(p=0.03)$, with the highest incidence in the group aged $61-70$ years $(35.7 \%)$, while the young adult group (31-40 years) developed one or more types of chronic post-amputation pain (14.3\%). There was no relation between the occurrence of post-amputation pain and gender, nationality, marital status, BMI, or educational level. However, there was a positive association between patient's complaints of comorbid diseases and the development of chronic post-amputation pain. Diabetes (95.6\%), though it was a common comorbid illness, did not have a statistically significant relation with the development of chronic post-amputation pain. Only 32 


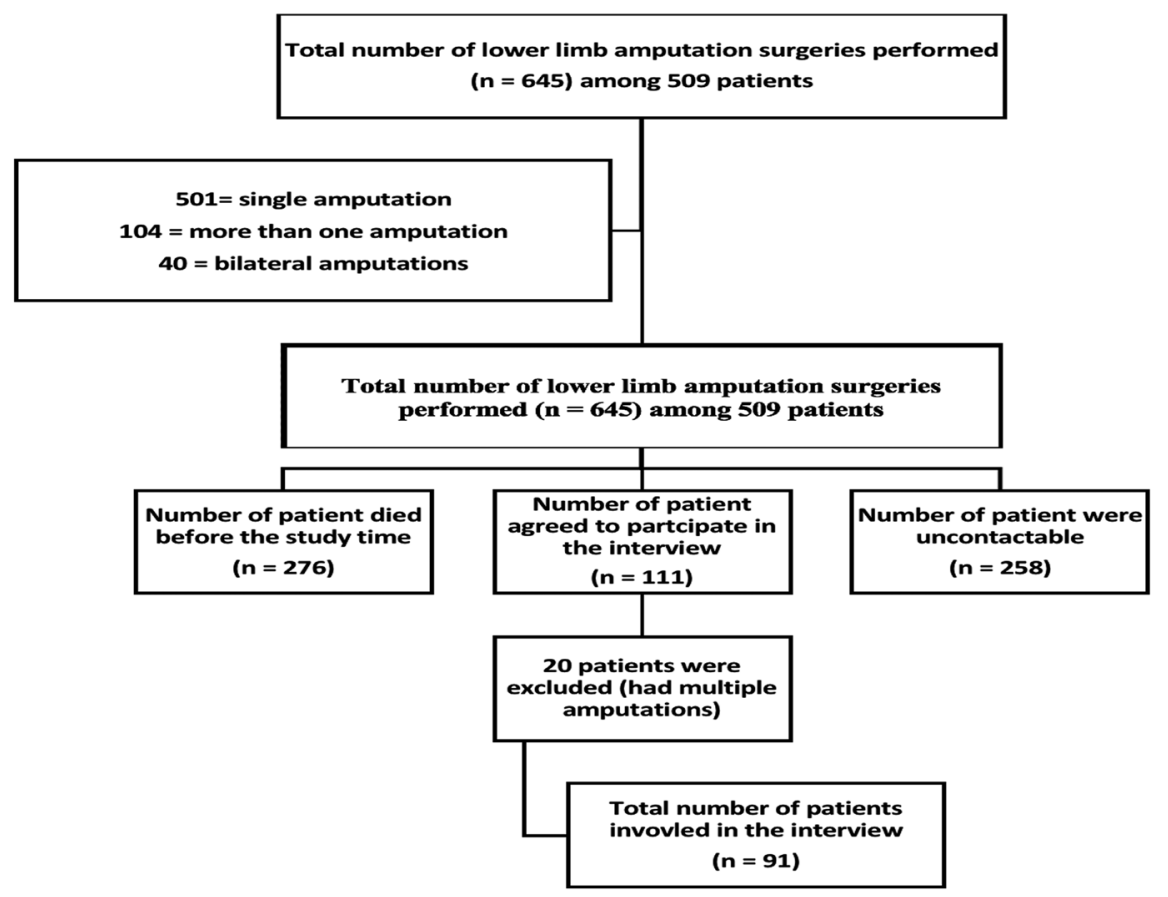

Figure 1 - STORB flow chart of participants.

out of 61 diabetic patients developed post-amputation pain, despite $67 \%$ of the 91 patients having their amputation due to diabetes complications and diabetic foot. Peripheral vascular disease (PVD) had a statistically significant association with the development of postamputation pain $(p=0.009)$, particularly PLP $(54.5 \%$; $p=0.016$; Table 2).

The number of people who were still employed after the amputation was $12(13.2 \%)$. Of the rest, 8 $(8.8 \%)$ were homemakers, and the rest were retired (23.1\%), unable to work (45.1\%), or unemployed but seeking jobs (9.9\%). Most of them (52.7\%) blamed their functional impairment on the disability resulting from amputation, while $6.6 \%$ said it was secondary to pain. The remaining $40.7 \%$ denied any functional impairment.

The overall ambulatory rate was $45 \%$, including $18.7 \%$ utilizing a prosthesis, and $15.4 \%$ depending on walking aids. There were $50(54.9 \%)$ bound to a wheelchair or bed. Surprisingly, 6 of the 12 bed-bound patients were of normal weight. There was no significant association between BMI and ambulatory status.

In all, $40.9 \%$ of PLP patients and $25 \%$ of RLP patients were referred to Physical therapy and Rehabilitation Department after their amputation. Such referral had no significant effect on the development or chronicity of post-amputation pain $(p=0.15)$.
Most of the amputation patients (71.4\%) experienced RLP with a duration of less than a year. Among them, $46.4 \%$ described the pain as moderate, and $50 \%$ stated that they experienced RLP a few times a day. Approximately $75 \%$ related its worsening to activity. Half of the patients (50\%) were able to sleep comfortably and $35.7 \%$ were awakened from their sleep by RLP. On the other hand, $14.3 \%$ could not sleep because of the pain. Approximately 50\% stated that RLP limited their ability to work, while $25 \%$ said it affected their leisure activities. Only $7.1 \%$ needed a caregiver, and $78.6 \%$ received treatment with equivocal satisfaction.

Residual limb pain was significantly associated with smoking, but it was unrelated to comorbid conditions, indications for amputation, intraoperative nerve ligation, or prosthesis use (Tables 384).

Regarding phantom pain, $13.3 \%$ started to have PLP within the rst 24 hours after the surgery, 27.3\% of patients developed PLP within one week after the amputation, and an equal number within 3 months of the amputation. There were $18.2 \%$ who developed PLP one year after the amputation.

Most patients (59.1\%) reported that their PLP was constant over time. Meanwhile, $36.4 \%$ reported that the pain decreased greatly. Only $4.5 \%$ had more pain as time passed, $31.8 \%$ experienced phantom pain a 
Post-amputation pain in Saudi Arabia ... AlMehman et al

Table 2 - Post amputation pain in relation to demographic, surgical, and comorbidity data $(\mathrm{N}=509)$.

\begin{tabular}{|c|c|c|c|c|c|c|}
\hline \multirow{3}{*}{ Variables } & \multicolumn{4}{|c|}{ Post amputation pain } & \multicolumn{2}{|c|}{ Likelihood ratio Chi-square } \\
\hline & \multicolumn{2}{|c|}{ No } & \multicolumn{2}{|c|}{ Yes } & \multirow{2}{*}{$\mathrm{X}^{2}$} & \multirow{2}{*}{$P$-value } \\
\hline & $\mathrm{n}$ & $\%$ & $\mathrm{n}$ & $\%$ & & \\
\hline \multicolumn{7}{|l|}{ Body mass index category } \\
\hline Underweight & 2 & 5.7 & 3 & 5.4 & \multirow{4}{*}{5.599} & \multirow{4}{*}{0.133} \\
\hline Normal weight & 11 & 31.4 & 31 & 55.4 & & \\
\hline Overweight & 11 & 31.4 & 13 & 23.2 & & \\
\hline Obese & 11 & 31.4 & 9 & 16.1 & & \\
\hline \multicolumn{7}{|l|}{ Smoking } \\
\hline Yes $^{\circ}$ & 5 & 14.3 & 11 & 19.6 & \multirow{3}{*}{1.006} & \multirow{3}{*}{0.605} \\
\hline No & 21 & 60.0 & 35 & 62.5 & & \\
\hline Ex-smoker & 9 & 25.7 & 10 & 17.9 & & \\
\hline \multicolumn{7}{|l|}{ Preoperative pain } \\
\hline Acute & 19 & 54.3 & 23 & 41.1 & \multirow{3}{*}{1.825} & \multirow{3}{*}{0.401} \\
\hline Chronic & 11 & 31.4 & 20 & 35.7 & & \\
\hline Do not know & 5 & 14.3 & 13 & 23.2 & & \\
\hline \multicolumn{7}{|l|}{ Reasons for amputation } \\
\hline PVD & 1 & 2.9 & 10 & 17.9 & \multirow{6}{*}{11.277} & \multirow{6}{*}{$0.046^{*}$} \\
\hline Acute ischemia & 1 & 2.9 & 5 & 8.9 & & \\
\hline Diabetic foot & 29 & 82.9 & 32 & 57.1 & & \\
\hline Infection & 4 & 11.4 & 6 & 10.7 & & \\
\hline Trauma & 0 & 0.0 & 2 & 3.6 & & \\
\hline Cancer & 0 & 0.0 & 1 & 1.8 & & \\
\hline Type of anesthesia & & & & & & \\
\hline General anesthesia & 28 & 80.0 & 44 & 78.6 & & \\
\hline Regional anesthesia & 4 & 11.4 & 8 & 14.3 & & \\
\hline MAC & 2 & 5.7 & 2 & 3.6 & 0.394 & 0.941 \\
\hline $\mathrm{GA}+\mathrm{PNB}$ & 1 & 2.9 & 2 & 3.6 & & \\
\hline Intraoperative nerve ligation & & & & & & \\
\hline No & 12 & 34.3 & 23 & 41.1 & & \\
\hline Yes & 8 & 22.9 & 16 & 28.6 & 1.470 & 0.480 \\
\hline Not applicable & 15 & 42.9 & 17 & 30.4 & & \\
\hline Level of amputation & & & & & & \\
\hline Toe & 12 & 34.3 & 9 & 16.1 & & \\
\hline Forefoot & 3 & 8.6 & 5 & 8.9 & & \\
\hline Foot & 1 & 2.9 & 2 & 3.6 & 4.070 & 0.397 \\
\hline BKA & 8 & 22.9 & 18 & 32.1 & & \\
\hline AKA & 11 & 31.4 & 22 & 39.3 & & \\
\hline Ambulation & & & & & & \\
\hline Prosthesis & 5 & 14.2 & 11 & 19.6 & 0.089 & 0.765 \\
\hline Walking aids & 10 & 28.6 & 9 & 16.1 & 1.993 & 0.158 \\
\hline Wheel-chair & 13 & 37.1 & 31 & 55.4 & 2.886 & 0.089 \\
\hline Bed-bound & 7 & 20.0 & 5 & 8.9 & 2.239 & 0.135 \\
\hline Comorbidity & & & & & & \\
\hline Hypertension & 23 & 65.7 & 29 & 51.8 & 1.724 & 0.189 \\
\hline Diabetes mellitus & 34 & 97.1 & 53 & 94.6 & 0.339 & 0.560 \\
\hline Heart disease & 8 & 22.9 & 23 & 41.1 & 3.283 & 0.070 \\
\hline Peripheral vascular disease & 6 & 17.1 & 24 & 42.9 & 6.822 & $0.009^{*}$ \\
\hline Coagulopathy & 3 & 8.6 & 0 & 0.0 & 5.898 & $0.015^{*}$ \\
\hline Rheumatic diseases & 0 & 0.0 & 0 & 0.0 & & \\
\hline Dyslipidemia & 6 & 17.1 & 14 & 25.0 & 0.795 & 0.373 \\
\hline Stroke & 6 & 17.1 & 6 & 10.7 & 0.760 & 0.383 \\
\hline Cancer & 0 & 0.0 & 0 & 0.0 & & \\
\hline Other & 11 & 31.4 & 11 & 19.6 & 1.604 & 0.205 \\
\hline
\end{tabular}


Table 3 - Residual limb pain characteristics ( $=509)$.

\begin{tabular}{|c|c|c|}
\hline Characteristics & $\mathrm{n}$ & $\%$ \\
\hline \multicolumn{3}{|l|}{ Residual limb pain } \\
\hline Yes & 28 & 30.8 \\
\hline No & 63 & 69.2 \\
\hline \multicolumn{3}{|l|}{ Duration } \\
\hline$<1$ year & 20 & 71.4 \\
\hline 2 years & 3 & 10.7 \\
\hline 3 years & 2 & 7.1 \\
\hline 4 years & 1 & 3.6 \\
\hline 5 years & 1 & 3.6 \\
\hline$>5$ years & 1 & 3.6 \\
\hline \multicolumn{3}{|l|}{ Severity } \\
\hline Mild & 4 & 14.3 \\
\hline Moderate & 13 & 46.4 \\
\hline Severe & 11 & 39.3 \\
\hline \multicolumn{3}{|l|}{ Frequency } \\
\hline Few times / month & 2 & 7.1 \\
\hline Few times / week & 8 & 28.6 \\
\hline Few times / day & 14 & 50.0 \\
\hline Constantly & 3 & 10.7 \\
\hline I don't know & 1 & 3.6 \\
\hline \multicolumn{3}{|l|}{ Worsening with physical activity } \\
\hline Strongly disagree & 1 & 3.6 \\
\hline Somewhat disagree & 1 & 3.6 \\
\hline Neither agree nor disagree & 3 & 10.7 \\
\hline Somewhat agree & 2 & 7.1 \\
\hline Strongly agree & 21 & 75.0 \\
\hline \multicolumn{3}{|l|}{ Activity limitations } \\
\hline No & 5 & 17.9 \\
\hline Limit leisure activities & 7 & 25.0 \\
\hline Limit work & 14 & 50.0 \\
\hline Need for caregiver & 2 & 7.1 \\
\hline \multicolumn{3}{|l|}{ Interference with sleep } \\
\hline Cannot sleep & 4 & 14.3 \\
\hline Wake up from sleep because of pain & 10 & 35.7 \\
\hline Sleep comfortably & 14 & 50.0 \\
\hline
\end{tabular}

few times a week, and $13.6 \%$ suffered constant PLP. Approximately 50\% reported pain lasting for seconds, while $40.9 \%$ said it would last for hours. There was no difference in terms of severity, with $36.4 \%$ experiencing severe phantom pain. For $27.3 \%$ the phantom pain originated from multiple sites; $45.5 \%$ of times at the toes followed by the legs (40.9\%), and less frequently from other sites.

Most patients (45.5\%) reported no limitation in activity due to PLP, while $18.2 \%$ reported the need for a caregiver. Approximately 50\% of PLP sufferers could sleep comfortably, and $31.8 \%$ reported inability to sleep. Among PLP sufferers, $45.5 \%$ had received medical treatment for PLP, and 60\% were satisfied with the treatment. No significant association was found between PLP and perioperative factors, ambulatory status, or prosthesis use (Tables $5 \& 6$ ).
Table 4 - Residual limb pain in relation to comorbidities and amputation.

\begin{tabular}{|c|c|c|c|c|c|c|}
\hline \multirow[b]{3}{*}{ Characteristics } & \multicolumn{4}{|c|}{ Residual limb pain } & \multirow{2}{*}{\multicolumn{2}{|c|}{$\begin{array}{c}\text { Likelihood ratio } \\
\text { Chi-square }\end{array}$}} \\
\hline & \multicolumn{2}{|c|}{ Yes } & \multicolumn{2}{|l|}{ No } & & \\
\hline & $\mathrm{n}$ & $\%$ & $\mathrm{n}$ & $\%$ & $\mathrm{X}^{2}$ & $P$-value \\
\hline \multicolumn{7}{|l|}{ Comorbidities } \\
\hline \multicolumn{7}{|l|}{ HTN } \\
\hline Yes & 13 & 46.4 & 39 & 61.9 & 1.885 & 0.170 \\
\hline No & 15 & 53.6 & 24 & 38.1 & & \\
\hline \multicolumn{7}{|l|}{$\mathrm{DM}$} \\
\hline Yes & 26 & 92.9 & 61 & 96.8 & 0.672 & 0.412 \\
\hline No & 2 & 7.1 & 2 & 3.2 & & \\
\hline \multicolumn{7}{|l|}{ HD } \\
\hline Yes & 12 & 42.9 & 19 & 30.2 & 1.367 & 0.242 \\
\hline No & 16 & 57.1 & 44 & 69.8 & & \\
\hline \multicolumn{7}{|l|}{ PVD } \\
\hline Yes & 12 & 42.9 & 18 & 28.6 & 1.753 & 0.185 \\
\hline No & 16 & 57.1 & 45 & 71.4 & & \\
\hline \multicolumn{7}{|l|}{ Coagulopathy } \\
\hline Yes & 0 & 0.0 & 3 & 4.8 & 2.252 & 0.133 \\
\hline No & 28 & 100.0 & 60 & 95.2 & & \\
\hline \multicolumn{7}{|l|}{ Dyslipidemia } \\
\hline Yes & 9 & 32.1 & 11 & 17.5 & 2.330 & 0.127 \\
\hline No & 19 & 67.9 & 52 & 82.5 & & \\
\hline \multicolumn{7}{|l|}{ Stroke } \\
\hline Yes & 5 & 17.9 & 7 & 11.1 & 0.737 & 0.391 \\
\hline No & 23 & 82.1 & 56 & 88.9 & & \\
\hline \multicolumn{7}{|l|}{ Smoking } \\
\hline Yes & 9 & 32.1 & 7 & 11.1 & & \\
\hline No & 15 & 53.6 & 41 & 65.1 & 4.667 & 0.031 \\
\hline Ex-smoker & 4 & 14.3 & 15 & 23.8 & & \\
\hline \multicolumn{7}{|c|}{ Reason for amputation } \\
\hline PVD & 6 & 21.4 & 5 & 7.9 & & \\
\hline Acute ischemia & 3 & 10.7 & 3 & 4.8 & & \\
\hline DM foot & 14 & 50.0 & 47 & 74.6 & 6.910 & 0.227 \\
\hline Infection & 4 & 14.3 & 6 & 9.5 & & \\
\hline Trauma & 1 & 3.6 & 1 & 1.6 & & \\
\hline Cancer & 0 & 0.0 & 1 & 1.6 & & \\
\hline \multicolumn{7}{|l|}{ Level of procedure } \\
\hline Toe & 6 & 21.4 & 15 & 23.8 & & \\
\hline Forefoot & 3 & 10.7 & 5 & 7.9 & & \\
\hline Foot & 2 & 7.1 & 1 & 1.6 & 2.037 & 0.729 \\
\hline BKA & 8 & 28.6 & 18 & 28.6 & & \\
\hline AKA & 9 & 32.1 & 24 & 38.1 & & \\
\hline \multicolumn{7}{|c|}{ Intraoperative nerve ligation } \\
\hline No & 9 & 32.1 & 26 & 41.3 & 0.699 & 0.705 \\
\hline Yes & 8 & 28.6 & 16 & 25.4 & & \\
\hline NA & 11 & 39.3 & 21 & 33.3 & & \\
\hline Use of prosthesis & 6 & 21.4 & 11 & 17.5 & 0.197 & 0.657 \\
\hline Total & 28 & 100.0 & 63 & 100.0 & & \\
\hline
\end{tabular}

HTN: hypertension, DM: diabetes mellitus, HD: heart disease, PVD: peripheral vascular disease, BKA: below knee amputation, AKA: above knee amputation, NA: not applicable 
Table 5 - Phantom limb pain characteristics.

\begin{tabular}{|c|c|c|}
\hline Characteristics & $\mathrm{n}$ & $\%$ \\
\hline \multicolumn{3}{|l|}{ Phantom limb pain } \\
\hline Yes & 22 & 24.2 \\
\hline No & 69 & 75.8 \\
\hline \multicolumn{3}{|l|}{ Onset } \\
\hline In the first 24 hours after amputation & 3 & 13.6 \\
\hline Within 1 week after the amputation & 6 & 27.3 \\
\hline Within 3 months after the amputation & 6 & 27.3 \\
\hline Within 6 months after the amputation & 1 & 4.5 \\
\hline Within 1 year after the amputation & 1 & 4.5 \\
\hline More than 1 year after the amputation & 4 & 18.2 \\
\hline I do not remember & 1 & 4.5 \\
\hline \multicolumn{3}{|l|}{ Course over time } \\
\hline Decrease greatly & 8 & 36.36 \\
\hline Remain the same & 13 & 59.1 \\
\hline Increase & 1 & 4.5 \\
\hline \multicolumn{3}{|l|}{ Frequency } \\
\hline a few times a year & 1 & 4.5 \\
\hline a few times a month & 3 & 13.6 \\
\hline a few times a week & 7 & 31.8 \\
\hline a few times a day & 4 & 18.2 \\
\hline a few times per hour & 4 & 18.2 \\
\hline always/constantly & 3 & 13.6 \\
\hline \multicolumn{3}{|l|}{ Duration } \\
\hline Seconds & 11 & 50.0 \\
\hline Hours & 9 & 40.9 \\
\hline Days & 1 & 4.5 \\
\hline Variable & 1 & 4.5 \\
\hline \multicolumn{3}{|l|}{ Severity } \\
\hline Mild & 7 & 31.8 \\
\hline Moderate & 7 & 31.8 \\
\hline Severe & 8 & 36.4 \\
\hline \multicolumn{3}{|l|}{ Phantom pain sensation origin } \\
\hline Ankle & 3 & 13.6 \\
\hline Foot & 5 & 22.7 \\
\hline Knee & 2 & 9.1 \\
\hline Leg & 9 & 40.9 \\
\hline Sole & 3 & 13.6 \\
\hline Thigh & 1 & 4.5 \\
\hline Toe & 10 & 45.5 \\
\hline Heel & 2 & 9.1 \\
\hline Whole leg & 1 & 4.5 \\
\hline Multiple sites & 6 & 27.3 \\
\hline \multicolumn{3}{|l|}{ Activity limitations } \\
\hline No & 10 & 45.5 \\
\hline Limits leisure activities & 5 & 22.7 \\
\hline Limits work & 3 & 13.6 \\
\hline Need for caregiver & 4 & 18.2 \\
\hline \multicolumn{3}{|l|}{ Interference with sleep } \\
\hline Cannot sleep & 7 & 31.8 \\
\hline Wake up from sleep because of pain & 4 & 18.2 \\
\hline Sleep comfortably & 11 & 50.0 \\
\hline
\end{tabular}

Table 6 - Phantom limb pain in relation to comorbidities, and amputation

\begin{tabular}{|c|c|c|c|c|c|}
\hline \multirow{2}{*}{ Characteristics } & & \multicolumn{2}{|c|}{ Phantom limb pain } & \multicolumn{2}{|c|}{$\begin{array}{c}\text { Likelihood ratio } \\
\text { Chi-square }\end{array}$} \\
\hline & & $\begin{array}{c}\text { Yes } \\
\text { n (\%) }\end{array}$ & $\begin{array}{c}\text { No } \\
\mathrm{n}(\%)\end{array}$ & $\mathrm{X}^{2}$ & $P$-value \\
\hline \multicolumn{6}{|l|}{ Comorbidities } \\
\hline \multirow[t]{2}{*}{ Hypertension } & Yes & $12(54.5)$ & $40(58.0)$ & \multirow{2}{*}{0.080} & \multirow{2}{*}{0.778} \\
\hline & No & $10(45.5)$ & $29(42.0)$ & & \\
\hline \multirow{2}{*}{$\begin{array}{l}\text { Diabetes } \\
\text { mellitus }\end{array}$} & Yes & $20(90.9)$ & 67 (97.1) & \multirow{2}{*}{1.309} & \multirow{2}{*}{0.253} \\
\hline & No & $2(9.1)$ & $2(2.9)$ & & \\
\hline \multirow[t]{2}{*}{ Heart disease } & Yes & $9(40.9)$ & $22(31.9)$ & \multirow{2}{*}{0.594} & \multirow{2}{*}{0.441} \\
\hline & No & $13(59.1)$ & $47(68.1)$ & & \\
\hline \multirow[t]{2}{*}{ PVD } & Yes & $12(54.5)$ & $18(26.1)$ & \multirow{2}{*}{5.854} & \multirow{2}{*}{$0.016^{*}$} \\
\hline & No & $10(45.5)$ & $51(73.9)$ & & \\
\hline \multirow[t]{2}{*}{ Coagulopathy } & Yes & $0(0.0)$ & $3(4.3)$ & 1693 & 0 193 \\
\hline & No & $22(100)$ & $66(95.7)$ & 1.693 & 0.193 \\
\hline Dyslipidemia & Yes & $5(22.7)$ & $15(21.7)$ & & \\
\hline & No & $17(77.3)$ & $54(78.3)$ & 0.009 & 0.923 \\
\hline Stroke & Yes & $3(13.6)$ & $9(13.0)$ & 0005 & 0943 \\
\hline & No & $19(86.4)$ & $60(87.0)$ & 0.005 & 0.943 \\
\hline Smoking & & & & & \\
\hline Yes & & $1(4.5)$ & $15(21.7)$ & & \\
\hline No & & $15(68.2)$ & $41(59.4)$ & 4.399 & 0.111 \\
\hline Ex-smoker & & $6(27.3)$ & $13(18.8)$ & & \\
\hline Preoperative pain & & & & & \\
\hline Acute & & $9(40.9)$ & $33(47.8)$ & & \\
\hline Chronic & & $7(31.8)$ & $24(34.8)$ & 0.987 & 0.611 \\
\hline Do not remember & & $6(27.3)$ & $12(17.4)$ & & \\
\hline Reason for amputati & & & & & \\
\hline PVD & & $7(31.8)$ & $4(5.8)$ & & \\
\hline Acute ischemia & & $1(4.5)$ & $5(7.2)$ & & \\
\hline DM foot & & $11(50.0)$ & $50(72.5)$ & 11026 & $0036 *$ \\
\hline Infection & & $2(9.1)$ & 8 (11.6) & 11.926 & 0.036 \\
\hline Trauma & & $1(4.5)$ & $1(1.4)$ & & \\
\hline Cancer & & $0(0.0)$ & $1(1.4)$ & & \\
\hline Level of procedure & & & & & \\
\hline Toe & & $2(9.1)$ & $19(27.5)$ & & \\
\hline Forefoot & & $3(13.6)$ & $5(7.2)$ & & \\
\hline Foot & & $0(0.0)$ & $3(4.3)$ & 8.152 & 0.086 \\
\hline BKA & & $5(22.7)$ & $21(30.4)$ & & \\
\hline AKA & & $12(54.5)$ & $21(30.4)$ & & \\
\hline Intraoperative nerve & ligat & & & & \\
\hline Not applicable & & $6(27.3)$ & $26(37.7)$ & & \\
\hline No & & $9(40.9)$ & $26(37.7)$ & 0.901 & 0.637 \\
\hline Yes & & $7(31.8)$ & $17(24.6)$ & & \\
\hline Sciatic & & $7(31.8)$ & $5(7.2)$ & 7.844 & $0.02^{*}$ \\
\hline Femoral & & $1(4.5)$ & $6(8.7)$ & 2.514 & 0.285 \\
\hline Type of anesthesia & & & & & \\
\hline General anesthesia & & $19(86.4)$ & $53(76.8)$ & 2.931 & 0.402 \\
\hline Regional anesthesi & & $2(9.1)$ & $10(14.5)$ & & \\
\hline MAC & & $0(0.0)$ & $4(5.8)$ & & \\
\hline $\mathrm{GA}+\mathrm{PNB}$ & & $1(4.5)$ & $2(2.9)$ & & \\
\hline Ambulation & & & & & \\
\hline Ambulatory & & $10(45.5)$ & $31(44.9)$ & 0.002 & 0.965 \\
\hline Prosthesis & & $6(27.3)$ & $11(15.9)$ & 1.322 & 0.250 \\
\hline Walking aids & & $4(18.2)$ & $15(21.7)$ & 0.131 & 0.718 \\
\hline Total & & $28(100)$ & $63(100)$ & & \\
\hline $\begin{array}{l}\text { PVD: peripheral v } \\
\text { above knee amputa } \\
\text { anesthesia, PNB: }\end{array}$ & 1 & $\begin{array}{l}\text { sease, BK- } \\
\text { AC: monit } \\
\text { al nerve bl }\end{array}$ & $\begin{array}{l}\text { elow knee } \\
\text { d anesthesi } \\
\text { zade, }{ }^{*} \text { stati }\end{array}$ & $\begin{array}{l}\text { putatic } \\
\text { are, GA } \\
\text { ally sig }\end{array}$ & $\begin{array}{l}\text { AKA: } \\
\text { reneral } \\
\text { icant }\end{array}$ \\
\hline
\end{tabular}


Of all patients suffering from PLP, RLP, or persistent postoperative neuropathic pain, only 24 (42.9\%) received medical treatment for pain management, $30.4 \%$ consulted their surgeons regarding their pain, and 2 underwent re-amputation of the amputation stump. Only $4(7.1 \%)$ consulted a pain specialist, and 3 had lumbar sympathetic block, with one patient proceeding with lumbar sympathectomy with pulsed radio frequency. Only one patient received local anesthetic with steroid injection at the stump and another one received an infusion of intravenous lidocaine. Of the patients suffering from PLP, 55.6\% had no coping method, and among of those with RLP, $42.9 \%$ had none. Reliance on analgesics was reported by $22.2 \%$ of those with PLP and $28.6 \%$ of those with RLP. None had tried tranquilizers or alcohol. Of the 24 who received medical treatment for phantom or RLP, 70.8\% used acetaminophen, $37.5 \%$ used anticonvulsant drugs,

$29.2 \%$ used non-steroidal anti-inflammatory drugs (NSAIDs), and $12.5 \%$ used antidepressants or opioids. Tramadol was not prescribed to any patient.

Discussion. The main finding of our study was the high frequency $(61.5 \%)$ of post-amputation pain among lower-limb amputees. This is similar to the findings. of other studies in which it ranged from $47-79 \% .4,7,8$ Persistent neuropathic pain was the most common type $(15.4 \%)$, followed by phantom pain $(11 \%)$, and RLP $(9.9 \%)$. The prevalences of all types of PAP pain were much lower than those reported among Western populations. ${ }^{3,4,8-12,17,20}$ In this study, the most common cause of functional impairment was disability (52.7\%) while only $6.6 \%$ considered PAP as a cause. Besides, $45 \%$ were mobile with a prosthesis or walking aid. This is opposite to the finding of Karns Schreuder et $\mathrm{al}^{21}$ who reported that lower-limb pain had a negative impact on the quality of life, particularly among post-amputation patients who have undergone prosthesis placement, while Davie-Smith et $\mathrm{al}^{22}$ in a systemic review found that the ability to walk with a prosthesis had a positive impact on quality of life. However, trans-femoral amputation has led to difficulty in using a prosthesis and in walking.

The average age of our patients was $57 \pm 12$ years, similar to previously published data from both the United States $(50.3 \pm 13.3$ years) and Singapore (62 years), while young and middle age were common age categories for amputation in other studies. ${ }^{7,9-11,23}$ Gallagher et al ${ }^{11}$ have reported that old age was associated with higher prevalence of severe PAP, while we could not find any correlation with age in our study. Although smoking was considered in many epidemiological studies to be a risk factor for amputation, a relation to amputation was not found in our study. ${ }^{24-26}$ Surprisingly, our study showed that smokers experienced less RLP when compared with non-smokers. The small sample size and the lack of information regarding the duration of smoking might have contributed to this finding. Further studies are needed to assess the relationship between smoking and the risk of amputation in Saudi Arabia. Most of our patients were males, but we could not detect any significant association between PAP and gender. Similarly, nationality, educational level, and obesity have no relation to the incidence of PAP. AlNozha et $\mathrm{al}^{27}$ epidemiological survey of 16917 Saudi patients revealed that diabetes was the most common among males than females, and this might help to explain the higher number of males in our sample. Further studies are needed to clarify these findings.

Peripheral vascular disease was associated with a high rate of amputation and PAP in this study. Finch et $\mathrm{al}^{28}$ reported the same finding in their study, where $30 \%$ of the PAP was the result of vascular insufficiency. Diabetes and PVD are coexisting morbidities of old age, which might contribute to this sequel.

Diabetic complications were the main reason for lower-limb amputation in our study. This is similar to the findings of previously published studies, in which diabetes and peripheral arterial disease were present in $75.6 \%$, while tumor and trauma were the main indications for lower-limb amputation among Caucasian patients. ${ }^{7-14,16,29}$ Similar to the findings of Gallagher et $\mathrm{al}^{11}$ in which AKA was associated with a higher incidence of PAP but the increase was not statistically significant. In our study, we found that preoperative pain had no correlation with the incidence of PAP, contrary to the findings of many other studies. ${ }^{14,30-33}$ The retrospective design of our study and its reliance on patient's memory might have led to this finding. Further studies are needed to test for this correlation among the local population.

The majority of those who had experienced RLP complained of pain for a period shorter than a year, and $46.4 \%$ had moderately severe pain. Approximately 50\% were able to sleep comfortably, while the remaining $50 \%$ complained of lack of sleep. Post-amputation pain and the impaired quality of life might lead to this disturbance of sleep, according to the findings of Yin et al. ${ }^{34}$

Although not perfectly illustrated due to the limited sample size, we do believe that an increase in the verbal analogue scale score for phantom pain was more commonly associated with amputations carried out above the level of the knee and those below the level of the ankle, as shown in prior studies in which PLP was 
more likely to occur with proximal amputations than with distal amputations. ${ }^{34,35-38}$ Proximal amputations are associated with more risk of delayed wound healing, infection, and development of stump pain. ${ }^{39}$

Managing PAP is a challenging situation, as many mechanisms were involved in the development of PAP. ${ }^{40}$ ere are many groups of medications recommended to treat PAP, paracetamol and NSAIDs are not commonly used among them. ${ }^{1}$ The high use of these medications might be related to the limited knowledge of the treating surgeons about the available medications, or the unfamiliarity of these surgeons regarding the proper treatment of neuropathic component of pain. Further studies are needed to assess the reasons behind improper treatment of PAP. Although pain clinics are specialized and experienced in dealing with such medical problems, the number of patients suffering from chronic PAP who have reported to the pain clinic was as low as 3.6\% among our patients. The complexity of PAP necessitates an interdisciplinary team that includes a pain specialist to look after the patients. The unfamiliarity of the treating doctors with pain specialty might contribute to the current situation. Further clinical trials are needed to assess the role of pain medicine in treating PAP.

Study limitations. The small number of patients agreed to participate in the interview as it might lead to enormously biased result (high nonresponse bias). In addition, the retrospective nature of the study and the reliance on patient's memories are other limitations of our study. Although our study provides good amount of information regarding PAP among the Saudi population, but we cannot generalize the results on other centers or cultures.

In conclusion, despite the small sample size, PAP is a major consequence of amputation with a significant impairment of the patient's life. Prospective welldesigned studies are needed to prevent the need for amputation and control PAP.

Acknowledgment. The authors gratefully acknowledge San Francisco Edit (www.sfedit.net) for English language editng.

\section{References}

1. Hsu E, Cohen SP. Postamputation pain: epidemiology, mechanisms, and treatment. J Pain Res 2013; 6: 121-136.

2. Grzebień A, Chabowski M, Malinowski M, Uchmanowicz I, Milan M, Janczak D. Analysis of selected factors determining quality of life in patients after lower limb amputation- a review article. Pol Przegl Chir 2017; 89: 57-61.

3. Stern JR, Wong CK, Yerovinkina M, Spindler SJ, See AS, Panjaki S, et al. A meta-analysis of long-term mortality and associated risk factors following lower extremity amputation. Ann Vasc Surg 2017; 42: 322-327.
4. Benzon H, Raja SN, Fishman S, Liu S, Cohen SP. Essentials of Pain Medicine 4th edition, In: Postamputation Pain. ELSEVIER; 2018. p 249.

5. Dillingham TR, Pezzin LE, MacKenzie EJ. Limb amputation and limb deficiency: epidemiology and recent trends in the United States. South Med J 2002; 95: 875-883.

6. Alotaibi A, Perry L, Gholizadeh L, Al-Ganmi A. Incidence and prevalence rates of diabetes mellitus in Saudi Arabia: an overview. J Epidemiol Glob Health 2017; 7: 211-218.

7. Ephraim PL, Wegener ST, MacKenzie EJ, Dillingham TR, Pezzin LE. Phantom pain, residual limb pain, and back pain in amputees: results of a national survey. Arch Phys Med Rehabil 2005; 86: 1910-1919.

8. Wartan SW, Hamann W, Wedley JR, McColl I. Phantom pain and sensation among British veteran amputees. Br J Anaesth 1997; 78: 652-659.

9. Smith DG, Ehde DM, Legro MW, Reiber GE, del Aguila M, Boone DA. Phantom limb, residual limb, and back pain after lower extremity amputations. Clin Orthop Relat Res 1999: 29-38.

10. Ehde DM, Czerniecki JM, Smith DG, Campbell KM, Edwards WT, Jensen MP, et al. Chronic phantom sensations, phantom pain, residual limb pain, and other regional pain after lower limb amputation. Arch Phys Med Rehabil 2000; 81: 1039-1044.

11. Gallagher P, Allen D, Maclachlan M. Phantom limb pain and residual limb pain following lower limb amputation: a descriptive analysis. Disabil Rehabil 2001; 23: 522-530.

12. Husum H, Resell K, Vorren G, Heng YV, Murad M, Gilbert M, et al. Chronic pain in land mine accident survivors in Cambodia and Kurdistan. Soc Sci Med 2002; 55: 1813-1816.

13. Ong BY, Arneja A, Ong EW. E ects of anesthesia on pain after lower-limb amputation. J Clin Anesth 2006; 18: 600-604.

14. Hanley MA, Jensen MP, Smith DG, Ehde DM, Edwards WT, Robinson LR. Preamputation pain and acute pain predict chronic pain after lower extremity amputation. J Pain 2007; 8: 102-109.

15. Richardson C, Glenn S, Nurmikko T, Horgan M. Incidence of phantom phenomena including phantom limb pain 6 months after major lower limb amputation in patients with peripheral vascular disease. Clin J Pain 2006; 22: 353-358.

16. Probstner D, uler LC, Ishikawa NM, Alvarenga RM. Phantom limb phenomena in cancer amputees. Pain Pract 2010; 10: 249-256.

17. Subedi B, Grossberg GT. Phantom limb pain: mechanisms and treatment approaches. Pain Res Treat 2011; 2011: 864605.

18. Kooijman CM, Dijkstra PU, Geertzen JHB, Elzinga A, van der Schans CP. Phantom pain and phantom sensations in upper limb amputees: an epidemiological study. Pain 2000; 87: 33-41.

19. Bouhassira D, Attal N, Alchaar H, Boureau F, Brochet B, Bruxelle J, et al. Comparison of pain syndromes associated with nervous or somatic lesions and development of a new neuropathic pain diagnostic questionnaire (DN4). Pain 2005; 114: 29-36.

20. Al-Wahbi AM. Impact of a diabetic foot care education program on lower limb amputation rate. Vasc Health Risk Manag 2010; 6: 923-934.

21. Krans-Schreuder HK, Bodde MI, Schrier E, Dijkstra PU, van den Dungen JA, den Dunnen WF, et al. Amputation for long-standing, therapy-resistant type-I complex regional pain syndrome. J Bone Joint Surg Am 2012; 94: 2263-2268. 
22. Davie-Smith F, Coulter E, Kennon B, Wyke S, Paul L. Factors influencing quality of life following lower limb amputation for peripheral arterial occlusive disease: a systematic review of the literature. Prosthet Orthot Int 2017; 41: 537-547.

23. Sin EI, Thong SY, Poon KH. Incidence of phantom limb phenomena after lower limb amputations in a Singapore tertiary hospital. Singapore Med J 2013; 54: 75-81.

24. Reiber GE. The epidemiology of diabetic foot problems. Diabet Med 1996; 13: S6-S11.

25. Qari FA, Akbar D. Diabetic foot: presentation and treatment. Saudi Med J 2000; 21: 443-446.

26. Liu M, Zhang W, Yan Z, Yuan X. Smoking increases the risk of diabetic foot amputation: a meta-analysis. Exp Ther Med 2018; 15: 1680-1685.

27. Al-Nozha MM, Al-Maatouq MA, Al-Mazrou YY, Al-Harthi SS, Arafah MR, Khalil MZ, et al. Diabetes mellitus in Saudi Arabia. Saudi Med J 2004; 25: 1603-1610.

28. Finch DR, Macdougal M, Tibbs DJ, Morris PJ. Amputation for vascular disease: the experience of a peripheral vascular unit. $\mathrm{Br}$ J Surg 1980; 67: 233-237.

29. Hussain MA, Al-Omran M, Salata K, Sivaswamy A, Forbes TL, Sattar N, et al. Population-based secular trends in lowerextremity amputation for diabetes and peripheral artery disease. CMAJ 2019; 191: E955-E961.

30. Nikolajsen L, Jensen TS. Phantom limb pain. Br J Anaesth 2001; 87: 107-116.

31. Flor H, Nikolajsen L, Staehelin Jensen T. Phantom limb pain: a case of maladaptive CNS plasticity? Nat Rev Neurosci 2006; 7: 873-881.
32. Nikolajsen L, Ilkjaer S, Krøner K, Christensen JH, Jensen TS. Thee in uence of preamputation pain on postamputation stump and phantom pain. Pain 1997; 72: 393-405.

33. Perkins FM, Kehlet H. Chronic pain as an outcome of surgery. A review of predictive factors. Anesthesiology 2000; 93: 1123-1133.

34. Yin Y, Zhang L, Xiao H, Wen CB, Dai YE, Yang G, et al. e pre-amputation pain and the postoperative dea erentation are the risk factors of phantom limb pain: a clinical survey in a sample of Chinese population. BMC Anesthesiol 2017; 17: 69.

35. Dijkstra PU, Geertzen JH, Stewart R, van der Schans CP. Phantom pain and risk factors: a multivariate analysis. $J$ Pain Symptom Manage 2002; 24: 578-585.

36. Weiser TG, Regenbogen SE, ompson KD, Haynes AB, Lipsitz SR, Berry WR, et al. An estimation of the global volume of surgery: a modelling strategy based on available data. Lancet 2008; 372: 139-144.

37. Manchikanti L, Singh V. Managing phantom pain. Pain Physician 2004; 7: 365-375.

38. Kelle B, Kozanoğlu E, Biçer ÖS, Tan İ. Association between phantom limb complex and the level of amputation in lower limb amputee. Acta Orthop Traumatol Turc 2017; 51: 142-145.

39. Stone PA, Flaherty SK, Aburahma AF, Hass SM, Jackson JM, Hayes JD, et al. Factors a ecting perioperative mortality and wound-related complications following major lower extremity amputations. Ann Vasc Surg 2006; 20: 209-216.

40. Woolf CJ. Pain: moving from symptom control toward mechanism-specific pharmacologic management. Ann Intern Med 2004; 140: 441-451. 